\title{
NOTA SOBRE LA ESTRUCTURA POBLACIONAL DE ECHINOCACTUS PLATYACANTHUS (CACTACEAE) EN LA RESERVA DE LA BIÓSFERA "BARRANCA DE METZTITLÁN", HIDALGO, MÉXICO
}

\author{
Malinali Castañeda-Romero', Monserrat Luna-Contreras ${ }^{1}$, Daniel Vela- \\ Godinez ${ }^{1}$, Gessel Montoya-Santiago ${ }^{1}$, Alejandro González-Bermúdez ${ }^{1}$, Rodrigo \\ Martínez Peña ${ }^{1}$, Manuel EsPerón-Rodríguez ${ }^{1,2,3}$
}
${ }^{1}$ Universidad Nacional Autónoma de México, Facultad de Ciencias, Licenciatura de Ciencias de la Tierra, 04510 México, D.F., México.
${ }^{2}$ Macquarie University, Faculty of Sciences and Engineering, Department of Biological Sciences, Sydney NSW 2109, Australia.
${ }^{3}$ Autor para la correspondencia: orcacomefoca@ciencias.unam.mx

\section{RESUMEN}

Echinocactus platyacanthus es uno de los cactus más abundantes y ampliamente distribuidos en México. En este trabajo se comparó la estructura poblacional de esta especie en dos áreas con grado de accesibilidad contrastante dentro de la Reserva de la Biósfera "Barranca de Metztitlán” en Hidalgo. El sitio de alcance difícil (S1) presentó un número más grande de individuos, con mayor altura y más organismos juveniles. Por su parte, la localidad con fácil acceso (S2) registró un predominio de adultos. Se censaron 456 plantas y solo se encontró una plántula. Se estimó una densidad de 1410 individuos/ha en S1 y únicamente 863 en S2. En ambos sitios se determinó el tipo de población como regresiva, ya que los individuos de la etapa de madurez se encontraron en un número significativamente más grande y la cantidad de los juveniles fue muy baja (12.9\%), hecho que sugiere un futuro decline poblacional.

Palabras clave: Barranca de Metztitlán, biznaga dulce o de barril, Echinocactus platyacanthus, población regresiva.

\section{ABSTRACT}

Echinocactus platyacanthus is one of the most abundant and widely distributed cacti in Mexico. In this work we compared the population structure of this species in two areas 
with contrasting accessibility degree within the Biosphere Reserve "Barranca de Metztitlán" in the state of Hidalgo. The site with difficult accessibility (S1) had a larger total number of individuals, with larger dimensions, and more juveniles. The site with easy accessibility (S2) had a predominance of adults. A total of 456 individuals were counted and only one sapling was found. We estimated a population density of 1410 individuals/ha in S1 and 863 individuals/ha in S2. We determined the type of population as regressive, because in both sites we found a significantly larger number of individuals in the stage of maturity and a low number of juveniles (12.9\%), which suggests a future population decline.

Key words: Barranca de Metztitlán, Echinocactus platyacanthus, regressive population, sweet or barrel biznaga.

\section{INTRODUCCIÓN}

La familia Cactaceae comprende cerca de 2000 especies en América. Con 913 taxa, México tiene la mayor riqueza en el mundo de estas plantas, y 80\% de las especies son endémicas al territorio de la República (Guzmán et al., 2003; JiménezSierra, 2011). Dentro de las regiones con mayor diversidad de sus representantes destacan en el centro de México el Valle de Tehuacán-Cuicatlán en Puebla, y la Reserva de la Biósfera "Barranca de Metztitlán" en Hidalgo. Esta reserva cuenta con 70 especies de cactáceas, con poblaciones importantes de Cephalocereus senilis, Neobuxbaumia polylopha, y varias biznagas, entre ellas Echinocactus platyacanthus (Semarnap, 1999).

Echinocactus platyacanthus, también conocida como biznaga dulce o de barril, es un cactus globular que puede alcanzar $2 \mathrm{~m}$ de altura y 1.2 de diámetro. Prospera en el matorral xerófilo (Jiménez-Sierra, 2011). Esta especie es endémica de México y se le encuentra entre los 1100 y 2400 m s.n.m. (Hernández y GómezHinostrosa, 2011). Su distribución es discontinua, aunque sus poblaciones no están muy aisladas (Hernández y Gómez-Hinostrosa, 2011). Se localiza en el desierto chihuahuense en Coahuila, Guanajuato, Hidalgo, Nuevo León, Querétaro, San Luis Potosí, Tamaulipas y Zacatecas. En el sur del Valle de Tehuacán, en Puebla y Oaxaca es relativamente común (Gómez-Hinostrosa y Hernández, 2000; Jiménez-Sierra y Eguiarte, 2010). Las áreas del norte y del sur están geográficamente aisladas por el Eje Neovolcánico Transversal (Trujillo Argueta, 1984).

La planta se encuentra protegida en México por la NOM-059-SEMARNAT-2010, bajo la categoría "sujeta a protección especial" (Semarnat, 2010), y está 
en la Lista Roja de la UICN en la categoría de "casi amenazada" (Hernández et al., 2013). Entre los riesgos que sufre la especie están i) la perturbación del suelo por parte del ganado, lo que provoca la pérdida de microhábitat adecuado para el establecimiento de nuevos individuos; ii) la explotación de sus poblaciones para su uso en la fabricación del dulce tradicional conocido como acitrón o biznaga; y iii) su uso como forraje vivo para cabras y burros (Jiménez-Sierra et al., 2007; Jiménez-Sierra y Eguiarte, 2010). Debido a que la planta tiene un crecimiento lento (Jiménez-Sierra y Eguiarte, 2010), la extracción de individuos adultos incrementa su vulnerabilidad.

Nuestro objetivo es generar datos sobre la estructura y densidad poblacional de Echinocactus platyacanthus dentro de la Reserva de la Biósfera "Barranca de Metztitlán" en dos localidades con diferente accesibilidad. En este trabajo evaluamos cómo afecta el impacto humano al crecimiento y la estructura poblacional de E. platyacanthus, ya que se considera que una de las principales amenazas que sufre es su extracción no controlada (Jiménez-Sierra y Eguiarte, 2010). Como indicador del impacto humano usamos la accesibilidad y la distancia entre los sitios donde se encuentra la especie y los asentamientos humanos, así como las carreteras más cercanas.

\section{MÉTODOS}

Área de estudio

La Reserva de la Biósfera "Barranca de Metztitlán" se encuentra en el estado de Hidalgo $\left(20^{\circ} 14^{\prime} 15^{\prime \prime}-20^{\circ} 45^{\prime} 26^{\prime \prime} \mathrm{N}\right.$ y $\left.98^{\circ} 23^{\prime} 00^{\prime \prime}-98^{\circ} 57^{\prime} 08^{\prime \prime} \mathrm{W}\right)$ y tiene una superficie de 96043 ha. La elevación en la reserva varía de 1200 a $2600 \mathrm{~m}$. El clima es seco (BShw) y semiseco (BS hw), causado por el efecto de sombra de lluvia de la Sierra Madre Oriental. La temperatura media oscila entre 18 y $22{ }^{\circ} \mathrm{C}$ y la precipitación anual es en promedio de $500 \mathrm{~mm}$ (Conanp, 2003).

\section{Métodos de muestreo}

El trabajo de campo se llevó a cabo el 27 y 28 de septiembre de 2014. Seleccionamos dos áreas con grado de accesibilidad contrastante. El sitio 1 (S1) tenía difícil acceso y una mayor distancia a la carretera y los asentamientos humanos, por lo que consideramos el S1 menos propicio a la explotación. El sitio 2 (S2) era de fácil alcance y se encontraba contiguo a los dos elementos antropógenas mencionados, lo que lo hace más susceptible para la extracción de las plantas. Las áreas muestreadas fueron $2000 \mathrm{~m}^{2}$ para S1 y $2015 \mathrm{~m}^{2}$ para S2. En cada sitio se censaron todos los individuos presentes y se midieron su altura y diámetro. 
Estructura poblacional

Encontramos ocho estados de desarrollo basados en el diámetro y la altura: plántulas, organismos juveniles 1 y 2, y organismos adultos 1-5 (Cuadro 1) (Jiménez-Sierra y Torres-Orozco, 2003; Jiménez-Sierra et al., 2007). La estructura poblacional se categorizó de acuerdo con el criterio de Hegland y Van Leeuwen (2001), Brys et al. (2003) y Aguraiuja et al. (2008), quienes señalan que las poblaciones "dinámicas" se caracterizan por una mayor proporción de organismos jóvenes (plántulas, juveniles e inmaduros), las "normales" (o estándar) presentan una cantidad más grande de adultos, pero con un alto número de plantas juveniles, y en las "regresivas" dominan los adultos, especialmente los de considerable tamaño con flores (en este tipo de población es difícil encontrar señales de rejuvenecimiento ya que hay un bajo número de individuos juveniles y plántulas).

Análisis estadístico

Se utilizó la prueba no paramétrica de Mann-Whitney $(P<0.05)$ para determinar si había diferencias significativas al comparar el diámetro y la altura entre sitios ( $\mathrm{S} 1$ y $\mathrm{S} 2)$. La prueba de Fisher $(\alpha=0.05)$ se usó para observar la misma categoría de distinciones en la estructura poblacional de ambos sitios. Desarrollamos modelos de regresión lineal para identificar la relación alométrica entre el diámetro y la altura en cada sitio, y la significancia de los modelos se verificó con una prueba de ANOVA. Se consideró la significancia estadística a 95\% para todos los casos. Los análisis estadísticos se realizaron con el software R versión 3.0.1 (R Core Team, 2014).

\section{RESULTADOS}

Encontramos diferencias al comparar el tamaño, la estructura poblacional y la relación alométrica (altura-diámetro) entre los sitios S1 y S2. Se censaron y midieron 456 individuos, 282 en el S1 y 174 en el S2. Solo se registró una plántula en el S2. Se hallaron adultos con flores y frutos en ambos sitios. Para el S1 se determinó una densidad de 1410 plantas/ha, mientras que en el S2 el valor fue de 863. En el S1 los individuos eran ligeramente más altos pero con menor anchura. El diámetro promedio en el S1 fue $54.53 \pm 27.09 \mathrm{~cm}$ y en el S2 de $64.35 \pm 33.01 \mathrm{~cm}$. En el S1 la altura media fue $76.0 \pm 38.98 \mathrm{~cm}$, y en el S2 de $71.79 \pm 39.87 \mathrm{~cm}$. Con respecto al diámetro encontramos diferencias significativas al comparar los sitios $(\mathrm{W}=28882, P=0.001)$; sin embargo, para la altura no se obtuvieron $(\mathrm{W}=21958$, $P=0.059)$. 
En lo que toca a la estructura poblacional, registramos que ésta fue significativamente diferente entre sitios $(P=0.031)$. Los estados de desarrollo con mayor número de individuos fueron adultos 2 y 3 , con diámetros entre 36 y $80 \mathrm{~cm}$. Después de las plántulas, los estados de desarrollo con menor cantidad de organismos fueron juveniles 1 , con mediciones entre 3 y $11 \mathrm{~cm}$, y adultos 5 , con diámetros superiores a $113 \mathrm{~cm}$ (Cuadro 1, Fig. 1).

En el S1 se censó una mayor cantidad de individuos en todos los estados de desarrollo, excepto plántulas y adultos 4 . En el S1, adultos 2 tuvo el máximo número de plantas, mientras que en el S2 superó adultos 3. Juveniles 2 tuvo la proporción más alta de individuos, seguido de adultos 1 .
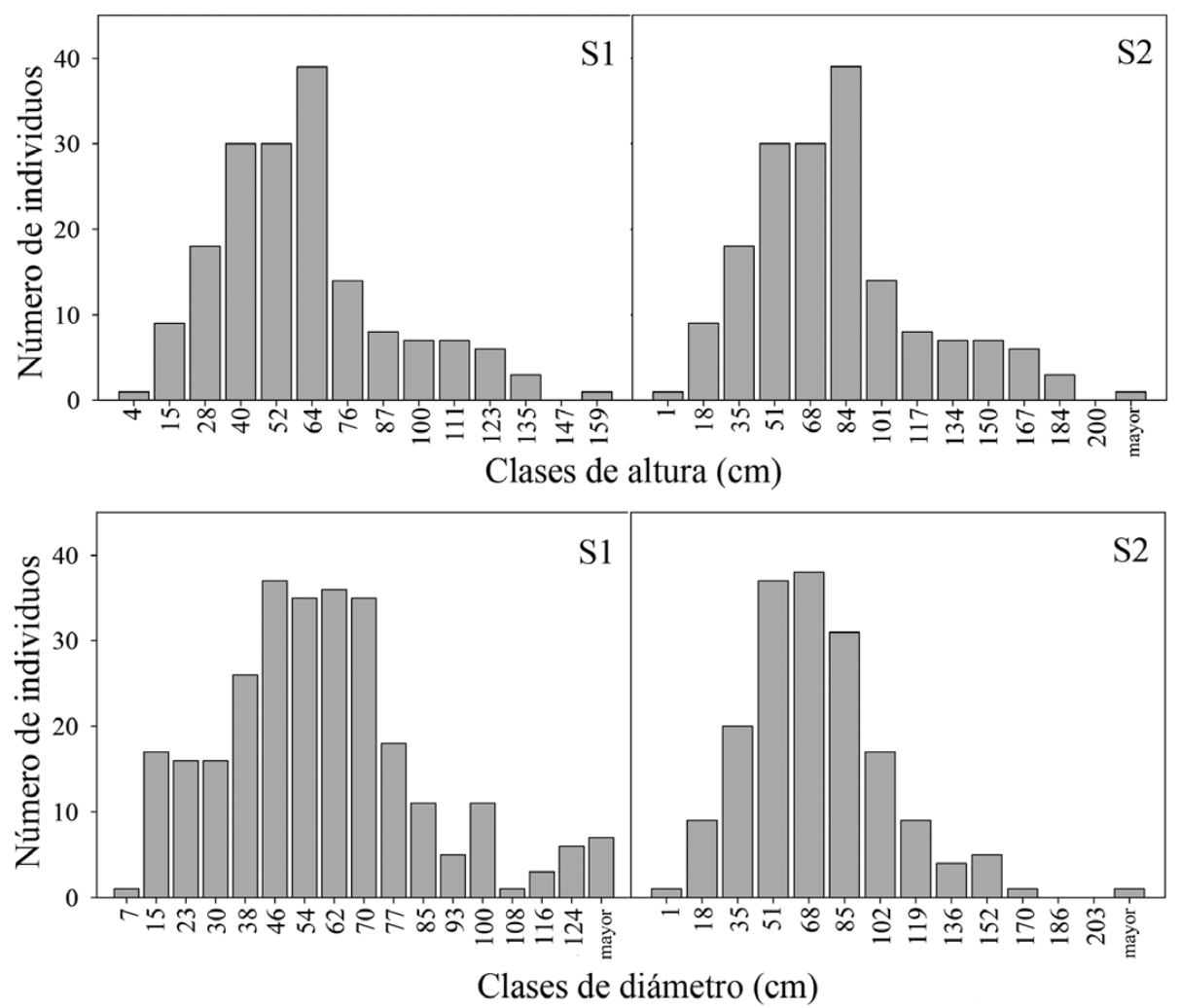

Fig. 1. Clases de tamaño de Echinocactus platyacanthus basadas en altura y diámetro. Se comparan dos sitios con diferente accesibilidad en la Reserva de la Biósfera "Barranca de Metztitlán", Hidalgo, México. El sitio 1 (S1) es de más difícil acceso y mayor distancia a los asentamientos humanos y a la carretera que el sitio 2 (S2). 
Cuadro 1. Número de individuos encontrados en cada estado de desarrollo de Echinocactus platyacanthus. Se comparan dos sitios con diferente accesibilidad en la Reserva de la Biósfera "Barranca de Metztitlán”, Hidalgo, México. El sitio 1 (S1) es de más difícil acceso y mayor distancia a los asentamientos humanos y a la carretera que el sitio 2 (S2). Los valores entre paréntesis indican las categorías de tamaño de diámetro en centímetros (sensu JiménezSierra y Torres-Orozco, 2003).

\begin{tabular}{lcccc}
\hline Sitio & $\begin{array}{c}\text { Plántula } \\
(<3)\end{array}$ & $\begin{array}{c}\text { Juvenil 1 } \\
(3-11)\end{array}$ & $\begin{array}{c}\text { Juvenil 2 } \\
(11.1-25)\end{array}$ & $\begin{array}{c}\text { Adulto 1 } \\
(25.1-36)\end{array}$ \\
\hline S1 & - & 8 & 34 & 30 \\
S2 & 1 & 4 & 12 & 14 \\
Total & 1 & 12 & 46 & 44 \\
\hline Sitio & Adulto 2 & Adulto 3 & Adulto 4 & Adulto 5 \\
& $(36.1-56)$ & $(56.1-80)$ & $(80.1-113)$ & $(>113.1)$ \\
\hline S1 & 91 & 78 & 26 & 15 \\
S2 & 47 & 50 & 33 & 13 \\
Total & 138 & 128 & 59 & 28 \\
\hline
\end{tabular}

Las poblaciones de ambos sitios fueron consideradas de tipo regresivo. Estuvieron compuestas fundamentalmente por un alto número adultos vegetativos, adultos con flores y sobre todo plantas generadoras (estados de desarrollo adultos 2-5), y una relativa escasez de plántulas, de las etapas de desarrollo prematuras y de juveniles (Cuadro 1). Los estadios juveniles tuvieron una baja representatividad con solo $12.9 \%$ de la población total.

Con respecto a la relación alométrica entre la altura y el diámetro encontramos que en el S1 ésta fue mayor (Altura = Diámetro $\left.(0.69)+38.21 ; \mathrm{R}^{2}=0.24\right)$ en comparación con el S2 (Altura $=$ Diámetro $(0.48)+40.42 ; \mathrm{R}^{2}=0.16$ ) (Fig. 2). Los modelos de regresión lineal para ambos sitios fueron significativos ( $\mathrm{S} 1: \mathrm{F}=90.282$, $P<0.001 ; \mathrm{S} 2: \mathrm{F}=33.435, P<0.001)$.

\section{DISCUSIÓN}

Encontramos diferencias entre los dos sitios con un posible distinto grado de impacto humano. Sin embargo, debido al bajo número de sitios, no tenemos evidencia suficiente para afirmar que las mismas se deban a la accesibilidad de los lugares, y a la distancia a los asentamientos humanos y a la carretera. Se requiere estudiar 
más localidades para poder determinar si estos factores afectan al crecimiento y a la estructura de las poblaciones de E. platyacanthus. Sin embargo, a pesar de no poder definir el origen de estas disimilitudes, registramos en el S1 una más grande cantidad de individuos con mayor altura.

El tipo de población recesiva encontrado en los sitios representa una amenaza para la especie, puesto que tiene un mayor riesgo de extinguirse en un futuro próximo (Aguraiuja et al., 2008), y al ser plantas de crecimiento lento (Vázquez-Sánchez et al., 2012), las poblaciones no pueden regenerarse de forma rápida e incorporar individuos con la misma velocidad con la que son extraídos. La presencia de una sola plántula pone en evidencia la vulnerabilidad de la especie, y la baja cantidad de organismos juveniles sugiere una futura disminución de la población. El reducido número de individuos juveniles y plántulas puede deberse al exceso de forraje causado por burros salvajes, ovejas y cabras (Jiménez-Sierra, 2011), cuyo efecto fue observado durante la realización de los censos.

Encontramos una mayor densidad de población en el S1 en comparación con las reportadas para otras áreas como en el Valle de Tehuacán-Cuicatlán, Puebla, (1016 individuos/ha; Jiménez-Sierra et al., 2007), y para otra región en Hidalgo (1111 individuos/ha; Jiménez-Sierra y Eguiarte, 2010). Para algunas localidades dentro del Valle de Tehuacán-Cuicatlán (Jiménez-Sierra et al., 2007) la densidad de población del S2 fue incluso mayor. En San Luis Potosí hay sitios en muy buen estado de conservación (Salas de León et al., 1999) con una densidad media estimada de 1500 individuos/ha (Goettsch, 2007).

Los datos demográficos obtenidos en este trabajo representan una contribución al conocimiento de la estructura de la población de E. platyacanthus dentro de la Reserva de la Biósfera "Barranca de Metztitlán". Esta especie requiere acciones de conservación debido al bajo número de plántulas y de individuos jóvenes. Si no se le da una protección apropiada, su existencia podría verse amenazada en el futuro. El estudio de la estructura de población y los datos sobre su tamaño son herramientas útiles para evaluar las prioridades de gestión y conservación, y para entender la dinámica regional en los casos en que se carece de datos sobre la distribución demográfica precisa (Aguraiuja et al., 2008).

\section{AGRADECIMIENTOS}

Los autores agradecen a Hugo Hernández, Karina López, Anahí Montiel, Rebeca Raygosa, y a los estudiantes de Ecología, grupo 1041, por su ayuda durante el 
trabajo de campo. También damos las gracias a Raúl Balderrama y Don Memo de "El Viejo Cactus" por su ayuda y asistencia durante el trabajo de campo. Agradecemos a la Facultad de Ciencias, de la Universidad Nacional Autónoma de México, por el apoyo recibido. El trabajo de campo realizado en este estudio no viola ninguna legislación ambiental de México. Los autores también agradecen a los revisores por sus comentarios críticos para el mejoramiento de este artículo.

\section{LITERATURA CITADA}

Aguraiuja, R., M. Zobel, K. Zobel y M. Moora M. 2008. Conservation of the endemic fern lineage Diellia (Aspleniaceae) on the Hawaiian Islands: can population structure indicate regional dynamics and endangering factors? Folia Geobot. 43: 3-18.

Brys, R., H. Jacquemyn, P. Endels, M. Hermy y G. De Blust. 2003. The relationship between reproductive success and demographic structure in remnant populations of Primula veris. Acta Oecol. 24(5): 247-253.

Conanp. 2003. Programa de Manejo Reserva de la Biosfera Barranca de Metztitlán, México. Comisión Nacional de Áreas Naturales. México, D.F., México. 202 pp.

Goettsch, B. 2007. Distribution modelling, macroecology and conservation: cacti of the Chihuahuan Desert Region. Ph.D. thesis. Animal and Plant Sciences, University of Sheffield, U.K.

Gómez-Hinostrosa, C. y H. M. Hernández. 2000. Diversity, geographical distribution, and conservation of Cactaceae in the Mier y Noriega region, Mexico. Biodivers Conserv. 9: 403-418.

Guzmán, U., S. Arias y P. Dávila. 2003. Catálogo de cactáceas mexicanas. Universidad Nacional Autónoma de México y Comisión Nacional para el Conocimiento y Uso de la Biodiversidad. México, D.F., México. 313 pp.

Hegland, S. J. y M. van Leeuwen. 2001. Population structure of Salvia pratensis in relation to vegetation and management of Dutch dry floodplain grasslands. J. Appl. Ecol. 38(6): 1277-1289.

Hernández, H. M. y C. Gómez-Hinostrosa. 2011. Mapping the cacti of Mexico. Their geographical distribution based on referenced records. In: David, D. (ed.). Succulent Plant Research 7: 1-128.

Hernández, H. M., M. Cházaro y C. Gómez-Hinostrosa. 2013. Echinocactus platyacanthus. The IUCN Red list of threatened species. Version 2014.3. www.iucnredlist.org. (consultado el 5 de diciembre de 2015).

Jiménez-Sierra, C. L. y R. Torres-Orozco. 2003. Estado actual de las poblaciones de la biznaga dulce Echinocactus platyacanthus (Cactaceae) en el SE de Puebla. ContactoS: 47: 28-34.

Jiménez-Sierra, C., M. C. Mandujano y L. E. Eguiarte. 2007. Are populations of the candy barrel cactus (Echinocactus platyacanthus) in the desert of Tehuacán, Mexico at risk? Population projection matrix and life table response analysis. Biol. Conserv. 135: 278-292. 
Jiménez-Sierra, C. y L. E. Eguiarte. 2010. Candy barrel cactus (Echinocactus platyacanthus Link \& Otto): a traditional plant resource in Mexico subject to uncontrolled extraction and browsing. Econ. Bot. 64: 99-108.

Jiménez-Sierra, C. 2011. Las cactáceas mexicanas y los riesgos que enfrentan. Rev. Dig. Universitaria 12(1): 1-23. http://www.revista.unam.mx/vol.12/num1/art04/art04.pdf. (consultado el 5 de diciembre de 2015).

R Core Team. 2014. R: A Language and environment for statistical computing. R Foundation for Statistical Computing, Vienna, Austria. http://www.R-project.org/. (consultado en enero de 2015).

Salas de Léon, S. N., A. García-Mendoza, J. A. Reyes Agüero y C. Villar Morales. 1999. Distribución geográfica y ecológica de la flora amenazada de extinción en la zona árida del estado de San Luis Potosí, México. Polibotánica 10: 1-21.

Semarnap. 1999. Documento técnico justificativo para la creación de la Reserva de la Biosfera Barranca de Metztitlán, Hidalgo. Secretaría de Medio Ambiente, Recursos Naturales y Pesca. México, D.F., México. 100 pp.

Semarnat. 2010. Norma Oficial Mexicana NOM-059-SEMARNAT-2010, Protección ambiental-Especies nativas de México de flora y fauna silvestres-Categorías de riesgo y especificaciones para su inclusión, exclusión o cambio-Lista de especies en riesgo. Diario Oficial de la Federación. 30 de diciembre 2010. Segunda Sección. México, D.F., México. pp. 77.

Trujillo Argueta, S. 1984. Distribucion geografica y ecologica de Echinocactus platyacanthus: un ejemplo de distribución disyunta. Cact. Suc. Mex. 29(4): 75-80.

Vázquez-Sánchez, M., T. Terrazas y S. Arias. 2012. El hábito y la forma de crecimiento en la tribu Cacteae (Cactaceae, Cactoideae). Bot. Sci. 90: 97-108.

Recibido en enero de 2015.

Reactivación en febrero de 2016.

Aceptado en marzo de 2016. 